\title{
A Case of Diffuse Large B-cell Lymphoma with Cardiac Involvement Incidently Diagnosed on Cardiac Imaging
}

\author{
Asif Husain Osmani and Irfan Maghfoor \\ Department of Oncology, King Faisal Specialist Hospital and Research Centre, Riyadh, Saudia Arabia
}

\begin{abstract}
Cardiac lymphomas, either primary or secondary, are mostly of B-cell lineage; among which, diffuse large B-cell lymphoma (DLBCL) is the most common. Secondary involvement of the heart is usually a late manifestation of disseminated lymphoma. Patients may be asymptomatic or may have non-specific symptoms, particularly when cardiac involvement is not extensive. A 22-year male patient presented with four months' history of progressive enlargement of left cervical lymph nodes with intermittent fever along with dysphagia and left ear pain.

Positron-emission tomography (PET)/computed tomography (CT) scan revealed abnormal fluorodeoxyglucose (FDG) uptake in multiple systemic locations including inferior wall of the myocardium. Subsequently, cardiac MRI showed left ventricular posterolateral wall abnormal area of delayed enhancement, corresponding to the FDG uptake on PET CT scan. Patient had remission after completion of standard chemotherapy protocol for the management of DLBCL with acceptable toxicity.
\end{abstract}

Key Words: Diffuse large B-cell lymphoma (DLBCL), Secondary, Heart, Chemotherapy.

How to cite this article: Osmani AH, Maghfoor I. A Case of Diffuse Large B-cell Lymphoma with Cardiac Involvement Incidently Diagnosed on Cardiac Imaging. J Coll Physicians Surg Pak 2021; 31(09):1102-1104.

\section{INTRODUCTION}

Cardiac lymphomas, either primary or secondary, are mostly of B-cell lineage; among which diffuse large B-cell lymphoma (DLBCL) is the most common. ${ }^{1}$ Despite the potentially lethal nature, most patients remain asymptomatic; hence, according to old reports, cardiac involvement from lymphoma constituted $18 \%$ in autopsy studies. ${ }^{2}$ Cardiac involvement by lymphoma poses a diagnostic challenge with lesions mimicking many other heartconditions. ${ }^{3}$

Herein, we present a case of a young man with stage IV DLBCL with secondary cardiac involvement, incidentally diagnosed on cardiacimaging.

\section{CASE REPORT}

A 22-year male patient presented with four months' history of progressive enlargement of left cervical lymph node mass, along with progressive dysphagia, left ear pain, and intermittent fever. Initially, patient was being managed as tonsillitis with antibiotics, which resulted in partial improvement of symptoms, but later the nodal mass increased in size and the patient underwenttonsillectomy.

Correspondence to: Dr. Asif Husain Osmani, Department of Oncology, King Faisal Specialist Hospital and Research Centre, Riyadh, Saudia Arabia

E-mail: osmaniasif77@gmail.com

Received: November 04, 2019; Revised: April 21, 2020;

Accepted: April 26, 2020

DOI: https://doi.org/10.29271/jcpsp.2021.09.1102
Histopathology of the tonsils revealed DLBCL, germinal centre cell subtype, with $B C L-2$ focal positive, $B C L-6$ positive, $C D 10$ positive, CD20 positive, CD3 negative, Ki-67 85\%, MUM-1 negative, CYCLIN-D1 negative, C-MYC weakly positive $>40 \%$, and EBV positive. He underwent fluorodeoxyglucose (FDG) positron-emission tomography (PET)/computed tomography (CT) for staging purpose. PET CT scan showed abnormal FDG uptake in multiple systemic organs, including nasopharynx, left tonsillar bed and the nodal mass in left neck, jejunum, ileum, incidental intramuscular focal FDG activity that corresponded to a hypodense lesion in the left biceps muscle, and abnormal focal FDG uptake seen in the inferior wall of the myocardium (Figure 1). Later, cardiac MRI revealed normal left ventricularsize with preserved systolicfunction $(E F=62 \%)$, along with left ventricular posterolateral wall abnormal area of delayed enhancement, corresponding to the FDG uptake on PET CT scan. The patient was planned for treatment with standard chemotherapy protocol for the management forDLBCL. During the pre-chemotherapy workup, the electrocardiogram (ECG), laboratory investigations, and bone marrow trephine were also normal. He was treated with $1^{\text {st }}$ cycle of chemotherapy R-CVP (rituximab, cyclophosphamide, vincristine, prednisone) for cytoreduction with CNS prophylaxis; and later cardiologist opinion was sought to evaluate for any contraindication to add anthracyclines to treatment regimen. The patientreceived threecycles of R-CHOP protocol underthesupervision of cardiologist with addition of doxorubicin. Interim PET CT scanfor response evaluation revealed completemetabolic remission (Figure 2). Later, patient completed six cycle of R-CHOP with 4 intra-thecal methotrexate to complete the planned treatment with no immediate complications. 


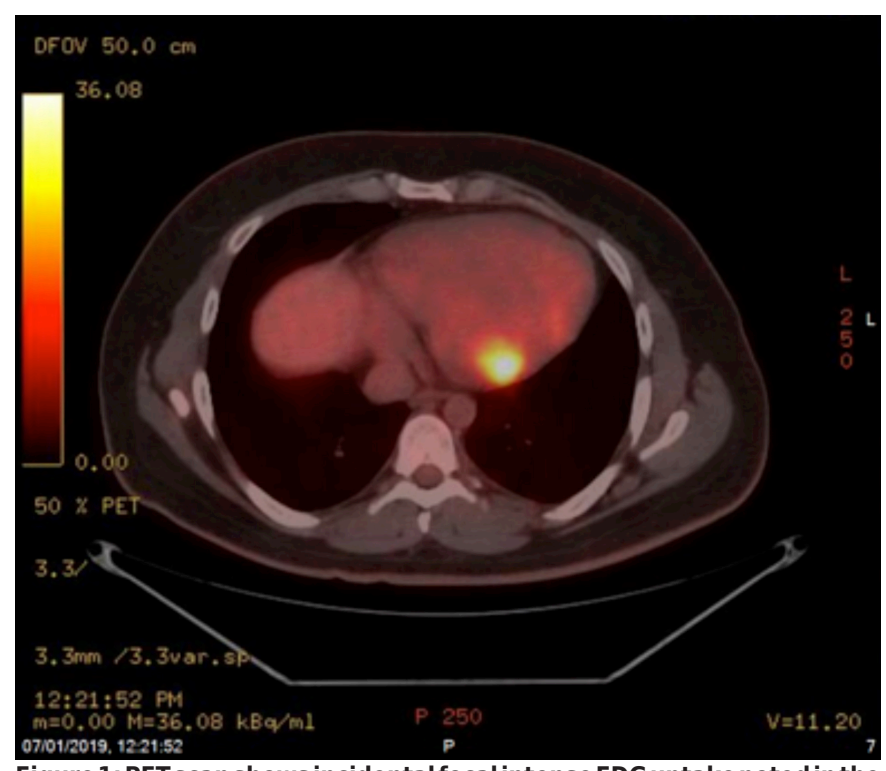

Figure 1: PET scan shows incidental focal intense FDG uptake noted in the inferior wall of the myocardium along with extra nodal disease on other sites.

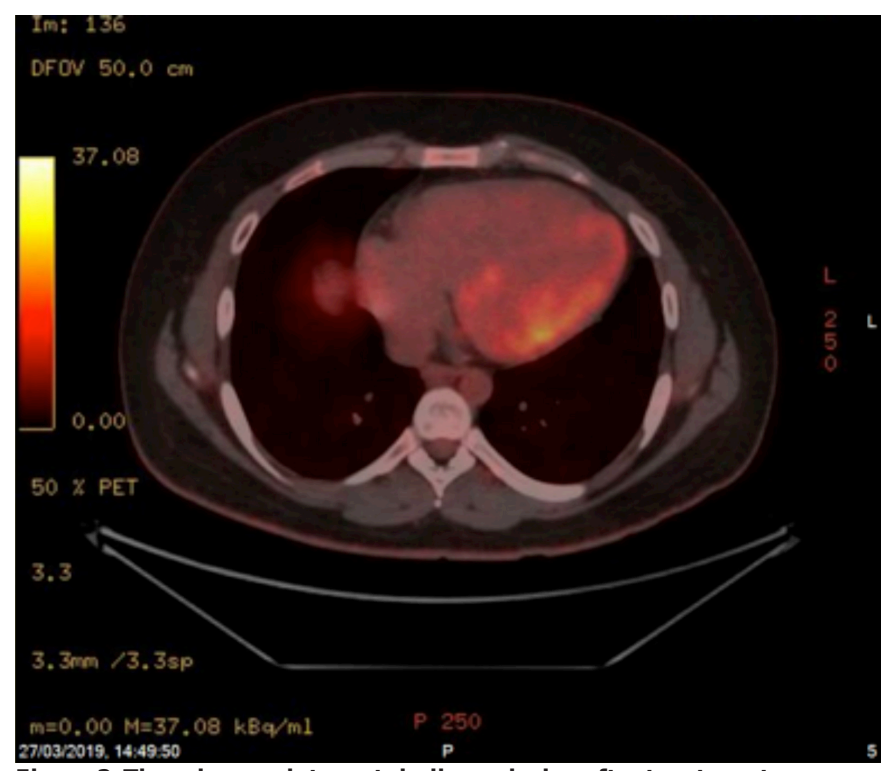

Figure 2: There is complete metabolic remission after treatment.

\section{DISCUSSION}

Secondary involvement of the heart by lymphoma is usually a late manifestation of disseminated disease. It may be asymptomatic or may have non-specific symptoms, particularly when themetastasis is notextensive. ${ }^{4}$ Therefore, it is frequently undetected before death. Most common sites of secondary cardiac involvement are left ventricle and right atrium in 55\% and 54\% of cases. ${ }^{5}$ It may occur through direct extension from the mediastinal disease, or hematogenous spread, and by retrograde lymphatic spread. The ECG and X-ray chest are neither sensitive nor specific for detecting the cardiac involvement. ${ }^{6}$ However, trans-thoracic ECG is considered as initial screening modality of choice. In a retrospective analysis of 29 patients with disseminated lymphoma, trans-thoracic ECG showed $60 \%$ sensitivity for detection of cardiac involvement. ${ }^{7}$ PET/ CT scan provides anatomical and functional imaging at the same position. ${ }^{8}$ It is often useful in differentiating DLBCL with secondary cardiac involvement from other types of cardiac tumours. ${ }^{9}$ Cardiac MRI is preferred imaging modality to evaluate the extent of pericardial and myocardial involvement by lymphoma. ${ }^{1,10}$ It has a superior tissue characterisation and; therefore, has higher sensitivity for detection of tumour infiltration. ${ }^{11}$ According to literature, the prognosis of lymphoma with secondary cardiac involvement.is unfavourable. ${ }^{12,13}$ However, improvements in survival have been observed in few reported cases. ${ }^{12}$ Generally, chemotherapy is mainstay of treatment for B-cell non-Hodgkin's lymphoma with secondary cardiac involvement, leading to prolonged median survival and durable remissions. ${ }^{12}$

\section{PATIENT'S CONSENT:}

Informed consent was obtained from the patient to publish the case data.

\section{CONFLICT OF INTEREST:}

The authors declared no conflict of interest.

\section{AUTHORS' CONTRIBUTION:}

AHO: Did literature search and drafted the manuscript.

IM: Conceived the idea and overall supervision.

\section{REFERENCES}

1. Jeudy J, Burke AP, Frazier AA. Cardiac lymphoma. Radiol Clin North Am 2016; 54(4): 689-710. doi: 10.1016/j.rcl.2016. 03.006.

2. Petersen CD, Robinson WA, Kurnick JE. Involvement of the heart and pericardium in the malignant lymphomas. Am J Med Sci 1976; 272(2):161-5. doi: 10.1097/00000441197609000-00005.

3. Sankaranarayanan R, Prasanna K. A case of primary cardiac lymphoma mimicking acute myocardial infarction. Clin Cardiol 2009; 32(8):E52-4. doi: 10.1002/clc.20441.

4. McDonnell PJ, Mann RB, Bulkley BH. Involvement of the heart by malignant lymphoma: A clinicopathologic study. Cancer 1982; 49(5):944 -51. doi: 10.1002/1097-0142 (19820301)49:5<944::aid-cncr2820490519>3.0.c0;2-c.

5. Travis WD BE, Müller-Hermelink HK, Harris C, eds. World health organization Classification of tumours. Pathology and genetics of tumours of the lung, pleura, thymus and heart. Lyon: IARC Press 2004; 282-4.

6. Faganello G, Belham M, Thaman R, Blundell J, Eller T, Wilde P. A case of primary cardiac lymphoma: Analysis of the role of echocardiography in early diagnosis. Echocardiography 2007; 24(8):889-92. doi: 10.1111/j.1540-8175.2007. 00472.x.

7. Palaskas N, Thompson K, Gladish G, Agha AM, Hassan S, Iliescu $C$, et al. Evaluation and management of cardiac tumors. Curr Treat Options Cardiovasc Med 2018; 20(4): 29. doi: 10.1007/s11936-018-0625-z.

8. Endo K, Oriuchi N, Higuchi T, lida Y, Hanaoka H, Miyakubo $\mathrm{M}$, et al. PET and PET/CT using 18F-FDG in the diagnosis and management of cancer patients. Int J Clin Oncol 2006; 11(4):286-96. doi: 10.1007/s10147-006-0595-0. 
9. Kikuchi Y, Oyama-Manabe N, Manabe O, Naya M, Ito YM, Hatanaka KC, et al. Imaging characteristics of cardiac dominant diffuse large B-cell lymphoma demonstrated with MDCT and PET/CT. Eur J Nucl Med Mol Imaging 2013; 40(9):1337-44. doi: 10.1007/s00259-013-2436-5.

10. Carter BW, Wu CC, Khorashadi L, Godoy MC, de Groot PM, Abbott GF, et al. Multimodality imaging of cardiothoracic lymphoma. Eur J Radiol 2014; 83(8): 1470-82. doi: 10.1016/j.ejrad.2014.05.018.

11. Lichtenberger JP, Dulberger AR, Gonzales PE, Bueno J,
Carter BW. MR imaging of cardiac masses. Top Magn Reson Imaging 2018; 27:103-111.

12. Gordon MJ, Danilova O, Spurgeon S, Danilov AV. Cardiac non-Hodgkin's lymphoma: clinical characteristics and trends in survival. Eur J Haematol 2016; 97(5):445-52. doi: 10.1111/ejh.12751.

13. Jeudy J, Kirsch J, Tavora F, Burke AP, Franks TJ, Mohammed $\mathrm{TL}$, et al. From the radiologic pathology archives: Cardiac lymphoma: Radiologic-pathologic correlation. Radiographics 2012; 32(5):1369-80. 